\title{
Energy averages and fluctuations in the decay out of superdeformed bands
}

\author{
A. J. Sargeant, ${ }^{1}$ M. S. Hussein, ${ }^{1}$ M. P. Pato, ${ }^{1}$ and M. Ueda ${ }^{2}$ \\ ${ }^{1}$ Nuclear Theory and Elementary Particle Phenomenology Group, Instituto de Física, Universidade de São Paulo, \\ Caixa Postal 66318, 05315-970 São Paulo, Brazil \\ ${ }^{2}$ Institute of Physics, University of Tsukuba, Ten-noudai 1-1-1, Tsukuba, Ibaraki, 305-8571, Japan
}

(Received 20 March 2002; published 4 December 2002)

\begin{abstract}
We derive analytic formulas for the energy average (including the energy average of the fluctuation contribution) and variance of the intraband decay intensity of a superdeformed band. Our results may be expressed in terms of three dimensionless variables: $\Gamma^{\downarrow} / \Gamma_{S}, \Gamma_{N} / d$, and $\Gamma_{N} /\left(\Gamma_{S}+\Gamma^{\downarrow}\right)$. Here $\Gamma^{\downarrow}$ is the spreading width for the mixing of a superdeformed (SD) state $|0\rangle$ with the normally deformed (ND) states $|Q\rangle$ whose spin is the same as $|0\rangle$ 's. The $|Q\rangle$ have mean lever spacing $d$ and mean electromagnetic decay width $\Gamma_{N}$ whilst $|0\rangle$ has electromagnetic decay width $\Gamma_{S}$. The average decay intensity may be expressed solely in terms of the variables $\Gamma^{\downarrow} / \Gamma_{S}$ and $\Gamma_{N} / d$ or, analogously to statistical nuclear reaction theory, in terms of the transmission coefficients $T_{0}(E)$ and $T_{N}$ describing transmission from the $|Q\rangle$ to the SD band via $|0\rangle$ and to lower ND states. The variance of the decay intensity, in analogy with Ericson's theory of cross section fluctuations, depends on an additional variable, the correlation length $\Gamma_{N} /\left(\Gamma_{S}+\Gamma^{\downarrow}\right)=(d / 2 \pi) T_{N} /\left(\Gamma_{S}+\Gamma^{\downarrow}\right)$. This suggests that analysis of an experimentally determined variance could yield the mean level spacing $d$ as does analysis of the cross section autocorrelation function in compound nucleus reactions. We compare our results with those of $\mathrm{Gu}$ and Weidenmüller.
\end{abstract}

DOI: 10.1103/PhysRevC.66.064301

PACS number(s): 21.60.-n, 24.60.-k

\section{INTRODUCTION}

The basic feature to be explained in the decay out of superdeformed (SD) rotational bands $[1-4]$ is that the intensity of the collective $\gamma$ rays emitted during the cascade down an SD band remains constant until a certain spin is reached whereafter it drops to zero within a few transitions. The sharp drop in intensity is believed to arise from mixing of the SD states with normally deformed (ND) states of identical spin [5]. The model of Refs. [6-9] attributes the suddenness of the decay out to the spin dependence of the barrier separating the SD and ND minima of the deformation potential. References [10-12] discuss the effect of the chaoticity of the ND states on the decay out.

In the present paper, we derive analytic formulas for the energy average (including the energy average of the fluctuation contribution) and variance of the intraband decay intensity of a superdeformed band in terms of variables which usefully describe the decay out $[3,4,13,14]$. We achieve this using the MIT approach to statistical nuclear reaction theory [15-17]. The MIT approach tackles calculation of the fluctuation cross section and other moments of the $S$ matrix by directly calculating averages of fluctuating functions of energy.

In agreement with Gu and Weidenmüller [13] (GW) we find that average of the total intraband decay intensity can be written as a function of the dimensionless variables $\Gamma^{\downarrow} / \Gamma_{S}$ and $\Gamma_{N} / d$, where $\Gamma^{\downarrow}$ is the spreading width for the mixing of an SD state with normally ND states of the same spin, $d$ is the mean level spacing of the latter and $\Gamma_{S}\left(\Gamma_{N}\right)$ are the electromagnetic decay widths of the SD (ND) states. Our formula for the variance of the total intraband decay intensity, in addition to the two dimensionless variables just mentioned, depends on the dimensionless variable $\Gamma_{N} /\left(\Gamma_{S}\right.$ $+\Gamma^{\downarrow}$ ). This additional variable is analogous to the correla- tion width of the Ericson's theory of cross section fluctuations. Its appearance suggests that measurement of the variance of the decay intensity could yield the mean level spacing $d$.

The paper is organized in the following manner. In Sec. II we express the average decay intensity as an average term plus the average of a fluctuation term. In Sec. III the expression for the average decay intensity obtained in Sec. II is evaluated approximately. In Sec. IV we calculate the variance of the intensity within the same approximation scheme as Sec. III. In Sec. V we interpret our results by analogy with statistical nuclear reaction theory, expressing our results in terms of transmission coefficients and compare our results with those of GW. In particular we suggest a reason why GW did not observe the variable $\Gamma_{N} /\left(\Gamma_{S}+\Gamma^{\downarrow}\right)$ in their calculation of the variance. Finally in Sec. VI we make some concluding remarks including mention of the limitations of our results.

\section{ENERGY AVERAGES AND FLUCTUATIONS}

The total intraband decay intensity has the form $[12,13,18]$

$$
I_{\mathrm{in}}=\left(2 \pi \Gamma_{S}\right)^{-1} \int_{-\infty}^{\infty} d E\left|A_{00}(E)\right|^{2}
$$

where the intraband decay amplitude is given by

$$
A_{00}(E)=\gamma_{S}\langle\widetilde{0}|G(E)| 0\rangle \gamma_{S} .
$$

Here $\gamma_{S}$ is the electromagnetic decay amplitude of superdeformed state $|0\rangle$ defined such that $\Gamma_{S}=\gamma_{S}^{2}$. We assume that the amplitude feeding $|0\rangle$ is also given by $\gamma_{S}$. The total Green's function $G$ is given by 


$$
G(E)=(E-H)^{-1},
$$

where the full nuclear Hamiltonian is denoted by $H$ and has a non-Hermitian part which accounts for coupling to the electromagnetic field [see Eqs. (5), (18), and (19) below]. The tilde is used to indicate the dual state or adjoint [15] of $|0\rangle$.

In what follows we employ the optical background representation introduced Kawai, Kerman, and McVoy [16]. These authors investigated fluctuation cross sections using a representation of the $S$ matrix in which the background $S$ matrix is chosen to be the energy average of the $S$ matrix itself, that is, the $S$ matrix corresponding to the optical potential. Here we use the same idea to decompose the decay amplitude, Eq. (2), into the sum of its Lorentzian energy average (Lorentzian energy averaging interval $I$ ),

$$
\overline{A_{00}(E)} \equiv A_{00}(E+i I / 2),
$$

plus a fluctuating part.

We proceed by introducing Feshbach's projection operators [15]

$$
P=|\widetilde{0}\rangle\langle 0| \quad \text { and } \quad Q=1-P .
$$

Let us introduce the notation $\mathcal{G}=P G P$ for the effective Green's function in the $P$ space and let $H_{P P}=P H P, H_{P Q}$ $=P H Q$, etc. Using the techniques of Ref. [16] we obtain

$$
\mathcal{G}=\overline{\mathcal{G}}+\mathcal{G}^{\mathrm{fl}}
$$

where the average effective Green's function $\overline{\mathcal{G}}=\mathcal{G}(E$ $+i I / 2)$ is given by

$$
\overline{\mathcal{G}}=[E-\overline{\mathcal{H}}]^{-1}
$$

and the average effective Hamiltonian is given by

$$
\overline{\mathcal{H}}=H_{P P}+\bar{W}_{P P},
$$

where coupling to the eliminated $Q$ space is accounted for by

$$
\bar{W}_{P P}=H_{P Q} \frac{1}{E-H_{Q Q}+i I / 2} H_{Q P}=\frac{-2 i}{I} V_{P Q} V_{Q P}
$$

and the energy dependent coupling potential $V$ introduced in Ref. [16] is defined by

$$
\begin{gathered}
V_{P Q}=H_{P Q} \sqrt{\frac{i I / 2}{E-H_{Q Q}+i I / 2}}, \\
V_{Q P}=\sqrt{\frac{i I / 2}{E-H_{Q Q}+i I / 2} H_{Q P} .}
\end{gathered}
$$

The fluctuating part of the effective Green's function $\mathcal{G}$ is given by

$$
\mathcal{G}^{\mathrm{fl}}=\overline{\mathcal{G}} V_{P Q} \frac{1}{E-H_{Q Q}-W_{Q Q}} V_{Q P} \overline{\mathcal{G}},
$$

where coupling back to the $P$ space is accounted for by

$$
W_{Q Q}=V_{Q P} \overline{\mathcal{G}} V_{P Q} .
$$

By construction $\overline{\mathcal{G}^{\mathrm{fl}}}=0$ in so much as $\overline{\mathcal{G}}$ is unchanged by reaveraging, that is if $\overline{\overline{\mathcal{G}}}=\overline{\mathcal{G}}$.

The corresponding decomposition of Eq. (2) for the transition amplitude is

$$
A_{00}(E)=\overline{A_{00}(E)}+A_{00}^{\mathrm{fl}}(E),
$$

where the energy average of the transition amplitude is

$$
\overline{A_{00}}=\gamma_{S} \overline{\mathcal{G}}_{00} \gamma_{S}
$$

and the fluctuating part of the transition amplitude is

$$
A_{00}^{\mathrm{fl}}=\gamma_{S} \mathcal{G}_{00}^{\mathrm{fl}} \gamma_{S} .
$$

Thus the average of Eq. (1) for the relative intensity may be written as the incoherent sum

$$
\overline{I_{\mathrm{in}}}=I_{\mathrm{in}}^{\mathrm{av}}+\overline{I_{\mathrm{in}}^{\mathrm{fl}}},
$$

where

$$
I_{\mathrm{in}}^{\mathrm{av}}=\overline{I_{\mathrm{in}}^{\mathrm{av}}}=\left(2 \pi \Gamma_{S}\right)^{-1} \int_{-\infty}^{\infty} d E\left|\overline{A_{00}(E)}\right|^{2}
$$

and

$$
\overline{I_{\mathrm{in}}^{\mathrm{fl}}}=\left(2 \pi \Gamma_{S}\right)^{-1} \int_{-\infty}^{\infty} d E \overline{\left|A_{00}^{\mathrm{fl}}(E)\right|^{2}}
$$

Up to this point no assumptions have been made except that the transition amplitude can be written in the form of Eq. (2). As will be made clear in Sec. III the meaning of this assumption is that $|0\rangle$ is a doorway for the decay from the SD band to the ND states and vice versa. In the manipulations subsequent to Eq. (2) we have put the average intensity in a form consisting of a background term coming from the smooth energy dependence of the doorway plus a term resulting from fluctuations on this background.

The representation we have used is particularly suitable for approximation when the ND states are overlapping. In Sec. III we evaluate Eqs. (15)-(17) for the average decay intensity $\overline{I_{\text {in }}}$ assuming that this is the case. In Sec. IV we calculate the variance which describes the way in which $I_{\text {in }}$ fluctuates about $\overline{I_{\text {in }}}$.

\section{AVERAGE DECAY INTENSITY}

Let us assume that $H_{P P}$ satisfies the eigenvalue equation

$$
H_{P P}|0\rangle=\left(E_{0}-i \Gamma_{S} / 2\right)|0\rangle
$$

and $H_{Q Q}$

$$
H_{Q Q}|Q\rangle=\left(E_{Q}-i \Gamma_{N} / 2\right)|Q\rangle .
$$


Here, $E_{0}$ denotes the energy of SD state $|0\rangle, \Gamma_{S}$ it's electromagnetic width for decay to the next lowest state in the SD band, $E_{Q}(Q=1, \ldots, N)$ the energy of the $N$ ND states $|Q\rangle$ with the same spin as $|0\rangle$ and $\Gamma_{N}$ the common electromagnetic width of the $|Q\rangle$ for decay to ND states of lower spin.

Further, let us write the matrix element of $W_{P P}$, Eq. (9), as

$$
\bar{W}_{00}=\left\langle 0\left|\bar{W}_{P P}\right| 0\right\rangle=\Delta^{\downarrow}-i \Gamma^{\downarrow} / 2 .
$$

Here, $\Delta^{\downarrow}=\operatorname{Re} \bar{W}_{00}$ is an energy shift that we ignore and $\Gamma^{\downarrow}$ $=-2 \operatorname{Im} \bar{W}_{00}$. Combining these definitions with Eqs. (7), (8), and (13) the average of the transition amplitude can be written as

$$
\overline{A_{00}}=\frac{\Gamma_{S}}{E-E_{0}+i\left(\Gamma_{S}+\Gamma^{\downarrow}\right) / 2} .
$$

We see that Eq. (21) exhibits the structure of an isolated doorway resonance. The doorway $|0\rangle$ has an escape width $\Gamma_{S}$ for decay to the SD state with next lower spin and a spreading width $\Gamma^{\downarrow}$ for decay to the ND states with the same spin which are reached by tunneling through the barrier separating the SD and ND wells. The doorway structure of Eq. (21) is due to the assumption that the transition amplitude can be written as in Eq. (2). The most general expression for the transition amplitude has the form $A_{a b}=\gamma_{a b}$ $+\Sigma_{c c^{\prime}} \gamma_{a c}\left\langle c|G| c^{\prime}\right\rangle \gamma_{c^{\prime} b}$, where $\gamma_{a c}$ describes the coupling of channels $a$ and $c$. In our doorway model the $c$ and $c^{\prime}$ stand for $|0\rangle$ or $|Q\rangle, Q=1, \ldots, N$, i.e., $\Sigma_{c}|c\rangle\langle c|=P+Q$, and $a$ and $b$ denote channels the (electromagnetic) coupling to which is taken is accounted for by the non-Hermitian part of $H$ [Eqs. (18) and (19)], that is they denote the SD state above $|0\rangle$, the SD below $|0\rangle$, and ND states whose spin is different from that of $|0\rangle$. The direct coupling of channels $a$ and $b$, $\gamma_{a b}$, is taken to be zero.

In order to evaluate $A_{00}^{\mathrm{fl}}$ it is useful to introduce eigenvectors and eigenvalues of the operator $H_{Q Q}+W_{Q Q}$ defined by

$$
\left(H_{Q Q}+W_{Q Q}\right)|q\rangle=\left(E_{q}-i \Gamma_{q} / 2\right)|q\rangle, \quad q=1, \ldots, N .
$$

Then from Eq. (14) and Eq. (10) for $\mathcal{G}^{\mathrm{fl}}$ we obtain

$$
\begin{aligned}
A_{00}^{\mathrm{fl}} & =\gamma_{S} \overline{\mathcal{G}}_{00} \sum_{q} \frac{\left\langle\widetilde{0}\left|V_{P Q}\right| q\right\rangle\left\langle\tilde{q}\left|V_{Q P}\right| 0\right\rangle}{E-E_{q}+i \Gamma_{q} / 2} \overline{\mathcal{G}}_{00} \gamma_{S} \\
& =\overline{A_{00}} \sum_{q} \frac{g_{0 q} g_{q 0} / \Gamma_{S}}{E-E_{q}+i \Gamma_{q} / 2} \overline{A_{00}},
\end{aligned}
$$

where

$$
\begin{aligned}
& g_{0 q}=\left\langle\widetilde{0}\left|V_{P Q}\right| q\right\rangle, \\
& g_{q 0}=\left\langle\widetilde{q}\left|V_{Q P}\right| 0\right\rangle .
\end{aligned}
$$

We now employ some statistical assumptions which are frequently used in statistical nuclear reaction theory [15] to derive an analytic formula for the decay intensity $\overline{I_{\text {in }}}$. We shall assume that the Lorentzian and box energy averages and the average over the label $q$ are all approximately equal, i.e., that for a suitable function $f_{q}(E)$ of $q$ and $E$,

$$
\begin{aligned}
\overline{f_{q}(E)}=f_{q}(E+i I / 2) & \approx \frac{1}{\Delta E} \int_{E_{0}+\Delta E / 2}^{E_{0}+\Delta E / 2} d E^{\prime} f_{q}\left(E^{\prime}\right) \\
& \approx \frac{1}{N} \sum_{q=1}^{N} f_{q}(E) .
\end{aligned}
$$

The width of the box average, $\Delta E$, is related to the width the Lorentzian energy average by $\Delta E \approx \pi I / 2$ and to the mean spacing $d$ of the $N$ ND states by $\Delta E \approx N d$. This approximation is good as long as $\Gamma / d \gg 1$ [see Eq. (39) below for the definition of $\Gamma]$. Within these assumptions we see from Eq. (9) that the $g_{0 q}$ and $g_{q 0}$ are related to $\bar{W}_{00}$ by

$$
\bar{W}_{00}=\frac{-2 i}{I} \sum_{q} g_{0 q} g_{q 0} \approx \frac{-\pi i}{\Delta E} \sum_{q} g_{0 q} g_{q 0} \approx \frac{-\pi i}{d} \overline{g_{0 q} g_{q 0}} .
$$

Thus the spreading with is given by

$$
\Gamma^{\downarrow}=\frac{2 \pi}{d} \operatorname{Re} \overline{g_{0 q} g_{q 0}}
$$

and the energy shift by

$$
\Delta^{\downarrow}=\frac{\pi}{d} \operatorname{Im} \overline{g_{0 q} g_{q 0}} .
$$

From Eq. (23) we can calculate the amplitude autocorrelation function

$$
\begin{aligned}
& \overline{A_{00}^{\mathrm{fl}}(E) A_{00}^{\mathrm{fl}}\left(E^{\prime}\right)^{*}}=\frac{1}{\Gamma_{S}^{2}} \overline{A_{00}(E)^{2}} \sum_{q q^{\prime}} \frac{g_{0 q} g_{q 0} g_{0 q^{\prime}}^{*} g_{q^{\prime} 0}^{*}}{\left(E-E_{q}+i \Gamma_{q} / 2\right)\left(E^{\prime}-E_{q^{\prime}}-i \Gamma_{q^{\prime}} / 2\right)} \overline{A_{00}\left(E^{\prime}\right)^{* 2}} \\
& =\frac{1}{\Gamma_{S}^{2}} \overline{A_{00}(\bar{\varepsilon}+\varepsilon / 2)^{2}} \sum_{q q^{\prime}} \frac{g_{0 q} g_{q 0} g_{0 q^{\prime}}^{*} g_{q^{\prime} 0}^{*}}{\left(\bar{\varepsilon}+\varepsilon / 2-E_{q}+i \Gamma_{q} / 2\right)\left(\bar{\varepsilon}-\varepsilon / 2-E_{q^{\prime}}-i \Gamma_{q^{\prime}} / 2\right)} \overline{A_{00}(\bar{\varepsilon}-\varepsilon / 2)^{* 2}} \text {, }
\end{aligned}
$$


where we have made the variable changes $\varepsilon=E-E^{\prime}$ and $\bar{\varepsilon}=\left(E+E^{\prime}\right) / 2$. Consider the middle factor in Eq. (31) which we anticipate is a function of $\varepsilon$ only

$$
a(\varepsilon)=\sum_{q q^{\prime}} \frac{g_{0 q} g_{q 0} g_{0 q^{\prime}}^{*} g_{q^{\prime} 0}^{*}}{\left(\bar{\varepsilon}+\varepsilon / 2-E_{q}+i \Gamma_{q} / 2\right)\left(\bar{\varepsilon}-\varepsilon / 2-E_{q^{\prime}}-i \Gamma_{q^{\prime}} / 2\right)} .
$$

We interpret the energy average in Eq. (32) to be an average over $\bar{\varepsilon}$. Employing a box energy average

$$
a(\varepsilon) \approx \frac{1}{\Delta E} \sum_{q q^{\prime}} \int_{E_{0}-\Delta E / 2}^{E_{0}+\Delta E / 2} d \bar{\varepsilon} \frac{g_{0 q} g_{q 0} g_{0 q^{\prime}}^{*} g_{q^{\prime} 0}^{*}}{\left(\bar{\varepsilon}+\varepsilon / 2-E_{q}+i \Gamma_{q} / 2\right)\left(\bar{\varepsilon}-\varepsilon / 2-E_{q^{\prime}}-i \Gamma_{q^{\prime}} / 2\right)} .
$$

If $\Delta E$ is large compared to the $\Gamma_{q}$ but small enough for the $E_{q}$ and $\Gamma_{q}$ to be treated as constants then we may extend the limits of integration to $\pm \infty$ and perform the integral using the calculus of residues to obtain

$$
a(\varepsilon) \approx \frac{2 \pi i}{\Delta E} \sum_{q q^{\prime}} \frac{g_{0 q} g_{q 0} g_{0 q^{\prime}}^{*} g_{q^{\prime} 0}^{*}}{\varepsilon+E_{q^{\prime}}-E_{q}+i\left(\Gamma_{q^{\prime}}+\Gamma_{q}\right) / 2} .
$$

Assuming that the phases of the $g_{0 q}$ and the $g_{q 0}$ are randomly distributed as a function of $q$ the double sum in Eq. (34) collapses to a single sum giving

$$
a(\varepsilon) \approx \frac{2 \pi i}{\Delta E} \sum_{q} \frac{\left|g_{0 q} g_{q 0}\right|^{2}}{\varepsilon+i \Gamma_{q}}
$$

Then employing the definition of the average given by Eq. (26)

$$
a(\varepsilon) \approx \frac{2 \pi i}{d} \overline{\left.\frac{\left|g_{0 q} g_{q 0}\right|^{2}}{\varepsilon+i \Gamma_{q}}\right]} .
$$

Assuming that the average of a ratio is equal to the ratio of the averages we get

$$
a(\varepsilon) \approx \frac{\Gamma^{\downarrow 2}}{2 \pi} \frac{i d}{\varepsilon+i \Gamma},
$$

with $\Gamma^{\downarrow}$ and $\Gamma$ introduced according to Eq. (38) and Eq. (39) below. The introduction of $\Gamma^{\downarrow}$ is based on the assumption that

$$
\overline{\left|g_{0 q} g_{q 0}\right|^{2}} \approx 2\left|\overline{g_{0 q} g_{q 0}}\right|^{2} \approx 2\left[\frac{\Gamma^{\downarrow} d}{2 \pi}\right]^{2} .
$$

The factor of 2 which appears in the first manipulation in Eq. (38) accounts for the self-correlation (present since the entrance and exit channel are both $|0\rangle$ ) and is equal to the elastic enhancement factor for compound elastic scattering in the overlapping resonance region. In the second manipulation in Eq. (38) we have again ignored the energy shift [see Eqs. (28) and (29)].

Equation (37) also introduces the correlation width

$$
\begin{aligned}
\Gamma & \approx \overline{\Gamma_{q}} \\
& =-2 \operatorname{Im} \overline{\left\langle\tilde{q}\left|H_{Q Q}+W_{Q Q}\right| q\right\rangle} \\
& =\Gamma_{N}+\Gamma^{\uparrow} \\
& \approx \Gamma_{N},
\end{aligned}
$$

where we used Eq. (19) for the electromagnetic width of the $|Q\rangle$ and introduced

$$
\begin{aligned}
\Gamma^{\uparrow}=-2 \operatorname{Im} \overline{\left\langle\tilde{q}\left|W_{Q Q}\right| q\right\rangle} & =-2 \operatorname{Im} \frac{\overline{g_{q 0} g_{0 q}}}{E-E_{0}+i\left(\Gamma_{S}+\Gamma^{\downarrow}\right) / 2} \\
& =\frac{\Gamma^{\downarrow} d}{2 \pi} \frac{\left(\Gamma_{S}+\Gamma^{\downarrow}\right)}{\left(E-E_{0}\right)^{2}+\left(\Gamma_{S}+\Gamma^{\downarrow}\right)^{2} / 4},
\end{aligned}
$$

which is the width for their decay back to $|0\rangle$. The approximations represented by Eqs. (39) and (42) will be discussed in Sec. V. Using Eq. (37) and approximation (42) we finally obtain

$$
\begin{aligned}
\overline{A_{00}^{\mathrm{fl}}(E) A_{00}^{\mathrm{fl}}\left(E^{\prime}\right)^{*}} \approx & 2\left(2 \pi \Gamma_{N} / d\right)^{-1}\left(\Gamma^{\downarrow} / \Gamma_{S}\right)^{2} \overline{A_{00}(E)^{2}} \\
& \times \frac{i \Gamma_{N}}{E-E^{\prime}+i \Gamma_{N}} \overline{A_{00}\left(E^{\prime}\right)^{* 2}} .
\end{aligned}
$$

When $E^{\prime}=E$ this reduces to

$$
\overline{\left|A_{00}^{\mathrm{fl}}\right|^{2}}=2\left(2 \pi \Gamma_{N} / d\right)^{-1} \frac{\Gamma_{S}^{2} \Gamma^{\downarrow 2}}{\left[\left(E-E_{0}\right)^{2}+\left(\Gamma_{S}+\Gamma^{\downarrow}\right)^{2} / 4\right]^{2}},
$$

which is the average of the fluctuation contribution to the transition intensity.

The integrals in Eqs. (16) and (17) for the average and fluctuation contributions to the total decay intensity may now be carried out using the calculus of residues. Substituting Eq. (21) into Eq. (16) we obtain

$$
I_{\mathrm{in}}^{\mathrm{av}}=\frac{1}{1+\Gamma^{\downarrow} / \Gamma_{S}} .
$$



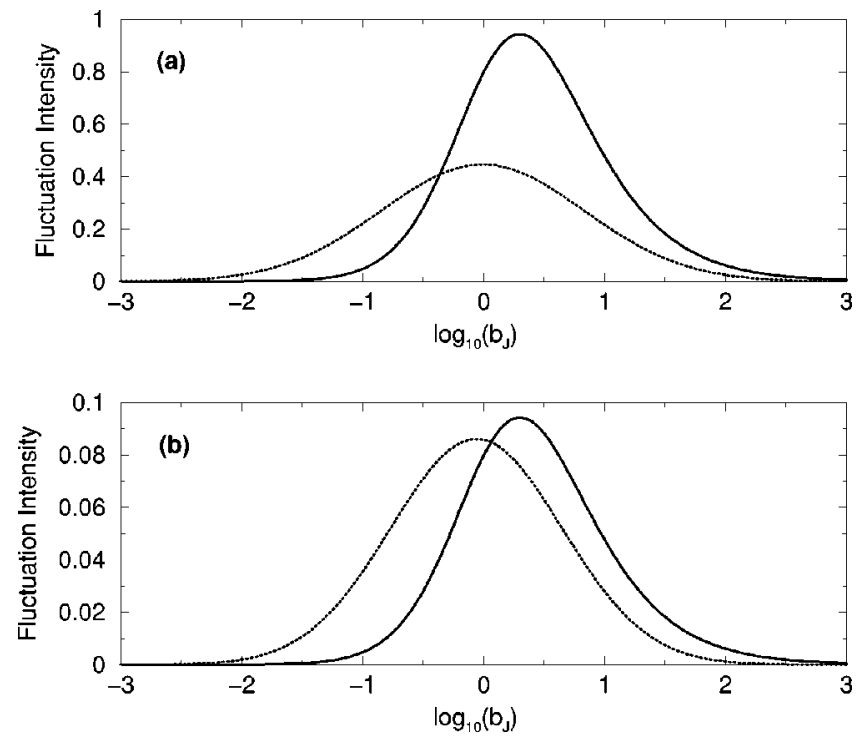

FIG. 1. Average of the fluctuation contribution to the intraband intensity $\overline{I_{\text {in }}^{\mathrm{fl}}}$ vs $\log _{10}\left(b_{J}\right)$, where $b_{J} \equiv \Gamma^{\downarrow} / \Gamma_{S}$. The solid lines were calculated using Eq. (47) and the dotted lines by GW's fit formula, Eq. (49). The variable $\Gamma_{N} / d$ took the value 0.1 for graph (a) and 1 for graph (b).

Equation (46) is identical with the equivalent result in GW (see also Ref. [14]). Substituting Eq. (45) into Eq. (17) we obtain

$$
\begin{aligned}
\overline{I_{\text {in }}^{\mathrm{fl}}} & =2\left(\pi \Gamma_{N} / d\right)^{-1} \frac{\left(\Gamma^{\downarrow} / \Gamma_{S}\right)^{2}}{\left(1+\Gamma^{\downarrow} / \Gamma_{S}\right)^{3}} \\
& =2\left(\pi \Gamma_{N} / d\right)^{-1} I_{\text {in }}^{\mathrm{av}}\left(1-I_{\text {in }}^{\mathrm{av}}\right)^{2}
\end{aligned}
$$

for the average fluctuation contribution to the average decay intensity.

Equation (47) for $\overline{I_{\text {in }}^{\mathrm{fl}}}$ is plotted in Fig. 1 and for comparison we have also plotted a fit formula which was obtained by GW,

$$
\begin{aligned}
\overline{I_{\text {in }}^{\mathrm{fl}}}= & {\left[1-0.9139\left(\Gamma_{N} / d\right)^{0.2172}\right] } \\
& \times \exp \left\{-\frac{\left[0.4343 \ln \left(\frac{\Gamma^{\downarrow}}{\Gamma_{S}}\right)-0.45\left(\frac{\Gamma_{N}}{d}\right)^{-0.1303}\right]^{2}}{\left(\Gamma_{N} / d\right)^{-0.1477}}\right\} .
\end{aligned}
$$

Qualitative agreement is seen between the two formulas. Note that Eq. (49) yields a negative intensity for $\Gamma_{N} / d$ $>1.51$ which excludes its use in this regime. Our result which is only strictly valid when $\Gamma_{N} / d \gg 1$ is simply inversely proportional to $\Gamma_{N} / d$. The exact result of GW for $\overline{I_{\text {in }}^{\mathrm{fl}}}$ [Eq. (24) in GW] which can be used for any $\Gamma_{N} / d$ also decreases monotonically with increasing $\Gamma_{N} / d$.

The dependence of $\overline{I_{\text {in }}^{\mathrm{fl}}}$ (and that of $I_{\text {in }}^{\text {av }}$ ) on $\Gamma^{\downarrow} / \Gamma_{S}$ results from the resonant doorway energy dependence of the decay amplitude $\overline{A_{00}(E)}$ [Eq. (21)]. This energy dependence also manifests itself in the average of the fluctuation contribution to the transition intensity $\overline{\left|A_{00}^{\mathrm{fl}}(E)\right|^{2}}$ [Eq. (45)]. GW include precisely the same energy dependence in their calculation by use of an energy dependent transmission coefficient to describe decay to the SD band [see Eq. (78) below and the discussion in Sec. V C]. This is the reason for our qualitative agreement with GW concerning $\overline{I_{\text {in }}}$.

\section{VARIANCE OF THE DECAY INTENSITY} by

The error incurred in making the energy average is given

$$
\Delta I_{\text {in }}=I_{\text {in }}-\overline{I_{\text {in }}}=I_{\text {in }}^{\mathrm{fl}}-\overline{I_{\text {in }}^{\mathrm{fl}}}+2 \operatorname{Re} \int_{-\infty}^{\infty} d E \overline{A_{00}(E)} A_{00}^{\mathrm{fl}}(E)^{*} .
$$

The average of the error vanishes: $\overline{\Delta I_{\text {in }}}=0$. A measure of the dispersion of the calculated $I_{\text {in }}$ is given by the variance

$$
\overline{\left(\Delta I_{\text {in }}\right)^{2}}=\overline{\left(I_{\text {in }}-\overline{I_{\text {in }}}\right)^{2}} .
$$

In order to evaluate $\overline{\left(\Delta I_{\text {in }}\right)^{2}}$ the averages indicated in Eq. (51) must be performed before the integration which appears in the definition of $I_{\text {in }}, E q$. (1). We obtain

$$
\begin{aligned}
\overline{\left(\Delta I_{\mathrm{in}}\right)^{2}} \approx & \left(2 \pi \Gamma_{S}\right)^{-2} \int_{-\infty}^{\infty} d E \int_{-\infty}^{\infty} d E^{\prime}\left\{\left|\overline{A_{00}^{\mathrm{fl}}(E) A_{00}^{\mathrm{fl}}\left(E^{\prime}\right)^{*}}\right|^{2}\right. \\
& \left.+2 \operatorname{Re} \overline{A_{00}(E)^{*}} \overline{A_{00}^{\mathrm{fl}}(E) A_{00}^{\mathrm{fl}}\left(E^{\prime}\right)^{*}} \overline{A_{00}\left(E^{\prime}\right)}\right\}
\end{aligned}
$$

In deriving Eq. (52) we have used $\overline{A_{00}^{\mathrm{fl}}(E)}$ $=\overline{A_{00}^{\mathrm{fl}}(E) A_{00}^{\mathrm{fl}}\left(E^{\prime}\right)}=0, \quad \overline{A_{00}^{\mathrm{fl}}(E) A_{00}^{\mathrm{fl}}\left(E^{\prime}\right)^{*}} \neq 0 \quad$ and $\overline{A_{00}^{\mathrm{fl}}(E) A_{00}^{\mathrm{fl}}(E) * A_{00}^{\mathrm{fl}}\left(E^{\prime}\right) A_{00}^{\mathrm{fl}}\left(E^{\prime}\right)^{*}}=\overline{\left|A_{00}^{\mathrm{fl}}(E)\right|^{2}} \overline{\left|A_{00}^{\mathrm{fl}}\left(E^{\prime}\right)\right|^{2}}$ $+\left|\overline{A_{00}^{\mathrm{fl}}(E) A_{00}^{\mathrm{fl}}\left(E^{\prime}\right)^{*}}\right|^{2}$ and we have assumed that averages of terms containing odd powers of $A_{00}^{\mathrm{fl}}$ vanish.

Substituting Eqs. (44) and (21) in Eq. (52), making the changes of integration variable

$$
\begin{gathered}
E-E_{0}=\left(\Gamma_{S}+\Gamma^{\downarrow}\right) x / 2, \\
E^{\prime}-E_{0}=\left(\Gamma_{S}+\Gamma^{\downarrow}\right) x^{\prime} / 2,
\end{gathered}
$$

and using Eq. (46) and (47) for $\overline{I_{\text {in }}^{\text {av }}}$ and $\overline{I_{\text {in }}^{\mathrm{fl}}}$ we are able to write $\overline{\left(\Delta I_{\text {in }}\right)^{2}}$ in the form

$$
\overline{\left(\Delta I_{\text {in }}\right)^{2}}=\overline{I_{\text {in }}^{\mathrm{fl}}} 2 f_{1}(\xi)+2 I_{\text {in }}^{\mathrm{av}} \overline{I_{\text {in }}^{\mathrm{fl}}} f_{2}(\xi),
$$

where the variable $\xi$ is defined by

$$
\begin{aligned}
\xi & \equiv \frac{\Gamma_{S}+\Gamma^{\downarrow}}{\Gamma_{N}} \\
& =\frac{\Gamma_{S}}{\Gamma_{N}}\left(1+\Gamma^{\downarrow} / \Gamma_{S}\right)=\frac{\Gamma_{S}}{\Gamma_{N}} I_{\text {in }}^{\mathrm{av}-1} \\
& =\frac{\Gamma^{\downarrow}}{\Gamma_{N}}\left(1+\Gamma_{S} / \Gamma^{\downarrow}\right)^{-1}=\frac{\Gamma^{\downarrow}}{\Gamma_{N}}\left(1-I_{\text {in }}^{\mathrm{av}}\right) .
\end{aligned}
$$


The functions $f_{1}$ and $f_{2}$ which have been introduced in Eq (55) are given by

$$
\begin{aligned}
f_{1}(\xi)= & \left(\frac{4}{\pi \xi}\right)^{2} \int_{-\infty}^{\infty} \frac{d x}{\left[x^{2}+1\right]^{2}} \\
& \times \int_{-\infty}^{\infty} \frac{d x^{\prime}}{\left[\left(x-x^{\prime}\right)^{2}+4 / \xi^{2}\right]\left[x^{\prime 2}+1\right]^{2}} \\
= & \frac{-8}{\pi^{2} \xi} \operatorname{Im} \int_{-\infty}^{\infty} \frac{d x}{\left[x^{2}+1\right]^{2}} \\
& \times \int_{-\infty}^{\infty} \frac{d x^{\prime}}{\left[x-x^{\prime}+2 i / \xi\right]\left[x^{\prime}+i\right]^{2}\left[x^{\prime}-i\right]^{2}} \\
= & \frac{-4}{\pi \xi} \operatorname{Im} \int_{-\infty}^{\infty} \frac{d x}{\left[x^{2}+1\right]^{2}}\left(\frac{1}{x+i(2 / \xi+1)}\right. \\
& \left.+\frac{i}{[x+i(2 / \xi+1)]^{2}}\right)
\end{aligned}
$$

and

$$
\begin{aligned}
f_{2}(\xi)= & \frac{4}{\pi^{2} \xi} \operatorname{Re} i \int_{-\infty}^{\infty} \frac{d x}{[x-i][x+i]^{2}} \\
& \times \int_{-\infty}^{\infty} \frac{d x^{\prime}}{\left[x-x^{\prime}+2 i / \xi\right]\left[x^{\prime}-i\right]^{2}\left[x^{\prime}+i\right]} \\
= & \frac{-2}{\pi \xi} \operatorname{Re} \int_{-\infty}^{\infty} \frac{d x}{[x-i][x+i]^{2}[x+i(2 / \xi+1)]} .
\end{aligned}
$$

Carrying out the second integrations in Eq. (60) and (62) we obtain

$$
f_{1}(\xi)=\frac{1}{(1+\xi)}+\frac{\xi}{(1+\xi)^{2}}+\frac{\xi^{2}}{2(1+\xi)^{3}}
$$

and

$$
f_{2}(\xi)=\frac{1}{2(1+\xi)} .
$$

The integrations in the calculation of $f_{1}$ and $f_{2}$ above were again carried out using the calculus of residues and were checked by numerical integration. The functions $f_{1}(\xi)$ and $f_{2}(\xi)$ are plotted in Fig. 2.

We have thus shown that a complete description of the decay out of a superdeformed band within the energy average approach requires three requires three dimensionless variables, $\Gamma^{\downarrow} / \Gamma_{S}, \Gamma_{N} / d$, and $\Gamma_{N} /\left(\Gamma_{S}+\Gamma^{\downarrow}\right)$. We find the following: (1) The average contribution of the background to the intraband decay intensity, $I_{\mathrm{in}}^{\mathrm{av}}$, [Eq. (46)] depends only on $\Gamma^{\downarrow} / \Gamma_{S}$. (2) The average of the fluctuation contribution, $\overline{I_{\mathrm{in}}^{\mathrm{Il}}}$, [Eq. (47)] depends on two variables: $\Gamma^{\downarrow} / \Gamma_{S}$ and $\Gamma_{N} / d$. (3)

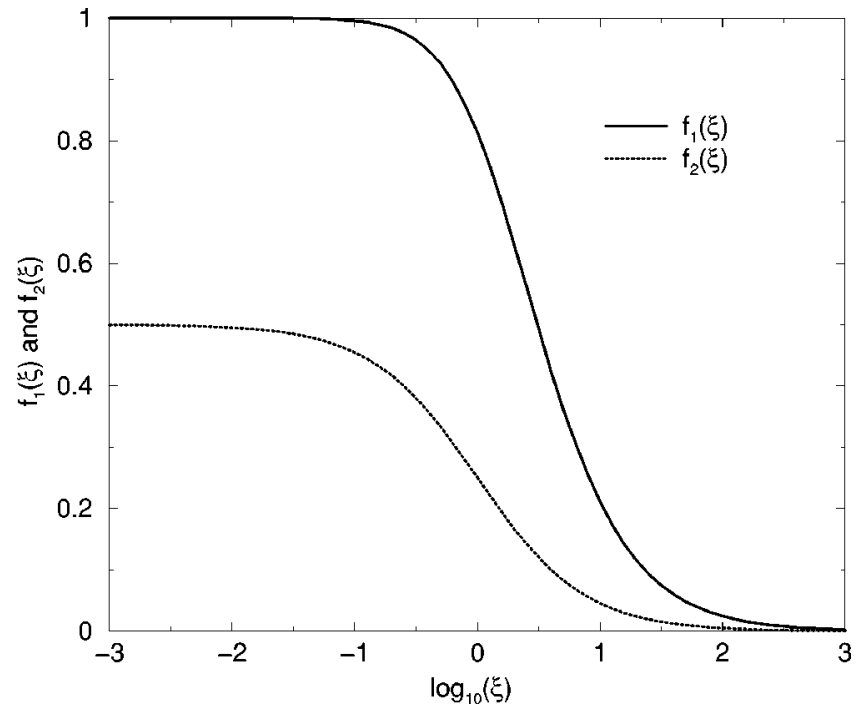

FIG. 2. The functions $f_{1}(\xi)$ [Eq. (63)] (solid line) and $f_{2}(\xi)$ [Eq. (64)] (dotted line) plotted vs $\log _{10}(\xi)$.

The variance, $\overline{\left(\Delta I_{\text {in }}\right)^{2}}$, [Eq. (55)] depends on three variables: $\Gamma^{\downarrow} / \Gamma_{S}, \Gamma_{N} / d$, and $\Gamma_{N} /\left(\Gamma_{S}+\Gamma^{\downarrow}\right)$.

Figure 3 shows a plot of the average intraband decay intensity $\overline{I_{\text {in }}}$ [Eq. (15)] calculated using Eqs. (46) and (47) for $I_{\text {in }}^{\text {av }}$ and $\overline{I_{\text {in }}^{\mathrm{fl}}}$. For comparison we also show the $\overline{I_{\text {in }}}$ that results when the GW fit formula [Eq. (49)] is used for $I_{\text {in }}^{\mathrm{H}}$ instead of

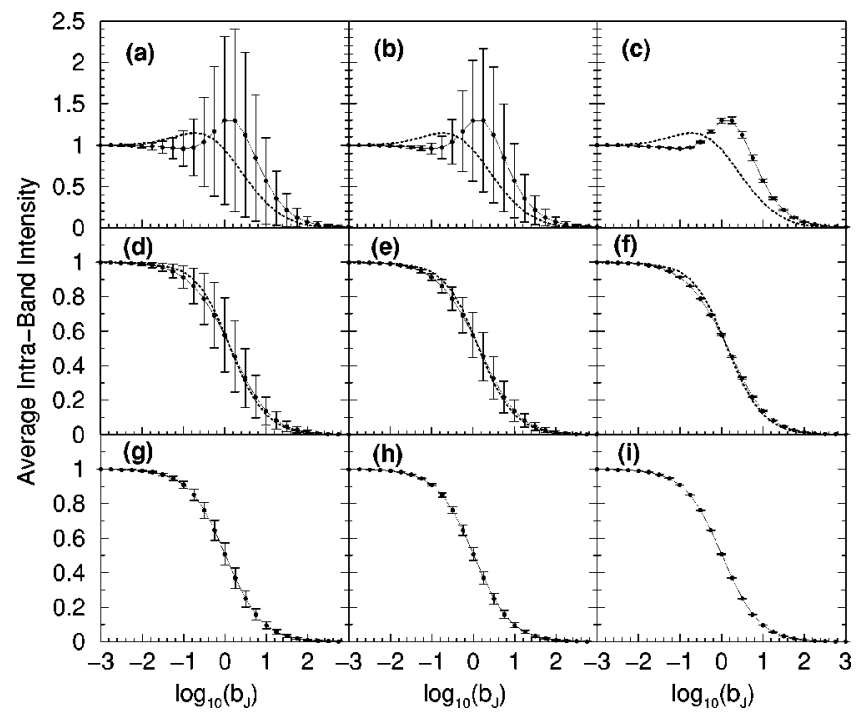

FIG. 3. Average intraband intensity $\overline{I_{\text {in }}}$ vs $\log _{10}\left(b_{J}\right)$ where $b_{J}$ $\equiv \Gamma^{\downarrow} / \Gamma_{S}$. The filled circles were calculated using Eq. (15) together with Eqs. (46) and (47). The error bars for the filled circles show $\sqrt{\overline{\left(\Delta I_{\text {in }}\right)^{2}}}$ calculated using Eq. (55) with $\xi$ in the form given by Eq. (58). The dotted lines were calculated in the same manner as the filled circles except that GW's fit formula, Eq. (49), was used in the place of Eq. (47). The variable $\Gamma_{N} / d$ took the following values: 0.1 in graphs (a), (b), and (c); 1 in graphs (d), (e), and (f); 10 in graphs (g), (h), and (i). The variable $\Gamma^{\downarrow} / \Gamma_{N}$ took the following values: $10^{-3}$ in graphs (a), (d), and (g); 1 in graphs (b), (e), and (h); $10^{3}$ in graphs (c), (f), and (i). 


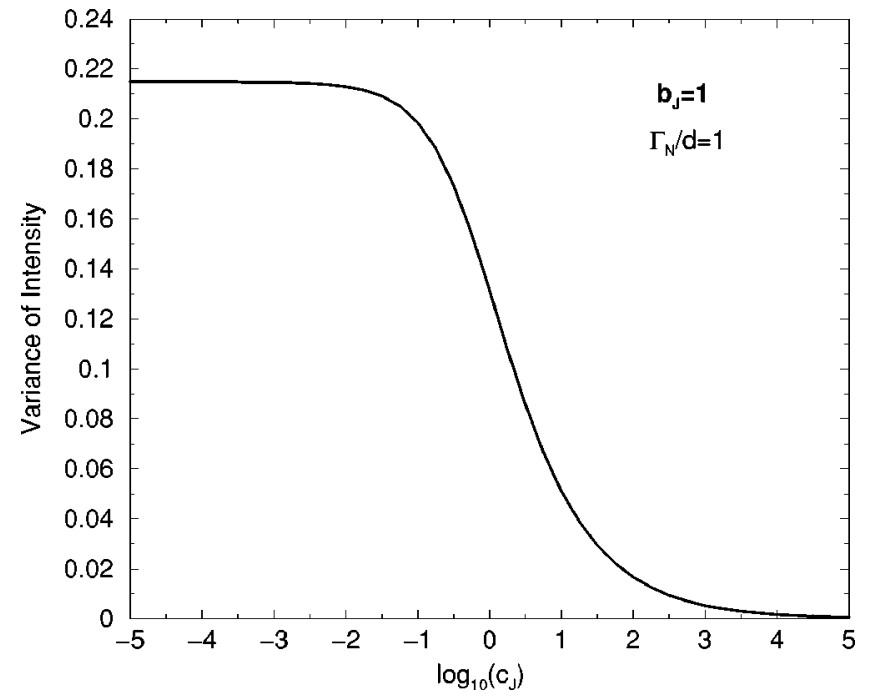

FIG. 4. The standard deviation of the decay intensity $\sqrt{\overline{\left(\Delta I_{\text {in }}\right)^{2}}}$ vs $\log _{10}\left(c_{J}\right)$, where $c_{J} \equiv \Gamma^{\downarrow} / \Gamma_{N}$ plotted using Eq. (55) with $\xi$ in the form given by Eq. (58) for fixed $b_{J}=\Gamma^{\downarrow} / \Gamma_{S}$ and $\Gamma_{N} / d$.

Eq. (47). The two curves are barely distinguishable for $\Gamma_{N} / d=1$. The $\mathrm{GW}$ fit formula incorrectly gives intensities that are greater than unity when $\Gamma_{N} / d=0.1$ as does our Eq. (47). The exact formula of GW [Eq. (24) in GW] does not suffer from this problem. Our results are only strictly valid when $\Gamma_{N} / d \gg 1$.

The error bars in Fig. 3 were calculated using Eq. (55) with $\xi$ in the form given by Eq. (58). We calculate $\sqrt{\overline{\left(\Delta I_{\text {in }}\right)^{2}}}$ for three values of the ratio $\Gamma^{\downarrow} / \Gamma_{N}$ (see figure caption). Since $\overline{\left(\Delta I_{\mathrm{in}}\right)^{2}}$ is proportional to $I_{\text {in }}^{\mathrm{fl}}$ it also increases monotonically to a maximum before decrease monotonically to zero as a function of $\Gamma^{\downarrow} / \Gamma_{S}$. For the same reason the error increases monotonically with decreasing $\Gamma_{N} / d$. The error estimate presented in GW exhibits the same trends with $\Gamma^{\downarrow} / \Gamma_{S}$ and $\Gamma_{N} / d$.

Since the variance depends only on $\left(\Gamma_{S}+\Gamma^{\downarrow}\right) / \Gamma_{N}$ in addition to $\Gamma^{\downarrow} / \Gamma_{S}$ and $\Gamma_{N} / d$, upon fixing the latter two variables the variance may be considered a function of any one of $\Gamma^{\downarrow} / \Gamma_{N}, \Gamma_{S} / \Gamma_{N}, \Gamma^{\downarrow} / d$, or $\Gamma_{S} / d$ [see Eqs. (57) and (58)]. In Fig. 3 we chose to use Eq. (58) for $\xi$ and fixed the value of $\Gamma^{\downarrow} / \Gamma_{N}$. When $\Gamma_{S} / \Gamma_{N}$ is fixed instead, a slightly different dependence on $\Gamma^{\downarrow} / \Gamma_{S}$ is obtained [compare Eqs. (57) and (58)]. Figure 4 shows a plot of the standard deviation, $\sqrt{\overline{\left(\Delta I_{\text {in }}\right)^{2}}}$, [Eq. (55)] as a function of $\Gamma^{\downarrow} / \Gamma_{N}$ for fixed $\Gamma^{\downarrow} / \Gamma_{S}$ and $\Gamma_{N} / d$. Ultimately, the variance like the intensity is a function of the spin of the decaying nucleus and could provide an additional probe to the spin dependence of the barrier separating the SD and ND wells which is contained in the spreading width $\Gamma^{\downarrow}$ [5-9].

Our result for the variance of the decay intensity $\overline{\left(\Delta I_{\text {in }}\right)^{2}}$ [Eq. (55)] has a structure reminiscent of Ericson's expression for the variance of the cross section [17]. This connection will be fully explored in the Sec. V. For now we note only that what distinguishes Eq. (55) from Ericson's expression for the variance of the cross section are the functions $f_{1}(\xi)$ and $f_{2}(\xi)$ which result from the energy integrations in Eq. (52).

\section{VARIANCE OF THE DECAY INTENSITY VERSUS AUTOCORRELATION FUNCTIONS OF STATISTICAL NUCLEAR REACTION THEORY}

\section{A. Results familiar from statistical nuclear reaction theory}

All moments of the $S$ matrix, $S_{a b}(E)$, the quantities that describe the way in which $S_{a b}(E)$ fluctuates about it's average, $\overline{S_{a b}(E)}$, can be expressed in terms of $\overline{S_{a b}(E)}$ itself [19]. Normally, specific moments such as the amplitude and cross section autocorrelation functions are expressed in terms of transmission coefficients, defined to be

$$
T_{a}=T_{a a}, \quad 0 \leqslant T_{a} \leqslant 1
$$

and their generalization

$$
T_{a b}=1-\sum_{c} \overline{S_{a c}} \overline{S_{b c}^{*}}=\sum_{c} \overline{S_{a c}^{\mathrm{fl}} S_{b c}^{\mathrm{fl}} *}
$$

Here $S_{a b}^{\mathrm{fl}}=S_{a b}-\overline{S_{a b}}$ is the fluctuating part of the $S$ matrix. The transmission coefficient $T_{a}$ is the probability of transmission from a compound nucleus resonance to channel $a$ and is obtained from the optical model.

In what follows we quote results for both the amplitude and cross section autocorrelation functions in the overlapping resonance region for the purpose of comparison with our results for the decay intensity. The amplitude autocorrelation function $c_{a b}\left(E, E^{\prime}\right)$ for amplitude

$$
A_{a b}=\delta_{a b}-i S_{a b}
$$

is defined by

$$
\begin{aligned}
c_{a b}\left(E, E^{\prime}\right) & =\overline{A_{a b}(E) A_{a b}\left(E^{\prime}\right)^{*}}-\overline{A_{a b}(E)} \overline{A_{a b}\left(E^{\prime}\right)^{*}} \\
& =\overline{A_{a b}^{\mathrm{fl}}(E) A_{a b}^{\mathrm{fl}}\left(E^{\prime}\right)^{*}} \\
& \approx \overline{\sigma_{a b}^{\mathrm{fl}}} \frac{i \Gamma}{E-E^{\prime}+i \Gamma} .
\end{aligned}
$$

The correlation width $\Gamma$ is given by

$$
\Gamma=\frac{d}{2 \pi} \sum_{a} T_{a} .
$$

Derivation of Eq. (70) requires the assumption that $\overline{A_{a b}(E)}$ $=\overline{A_{a b}\left(E^{\prime}\right)}$. This assumption together with statistical assumptions equivalent to those employed in our treatment of the factor $a(\varepsilon)$ [Eq. (32)] gives rise to a $c_{a b}\left(E, E^{\prime}\right)$ which depends solely on the difference of the two energies $E$ $-E^{\prime}$.

Equation (44), which is essentially $c_{00}\left(E, E^{\prime}\right)$ does not depend solely on $E-E^{\prime}$. The background energy modulation in both $E$ and $E^{\prime}$ which is characteristic of an isolated doorway resonance is explicit. In the present case it cannot be assumed that $\overline{A_{00}(E)} \neq \overline{A_{00}\left(E^{\prime}\right)}$ for arbitrary $E$ and $E^{\prime}$. The double integral for the variance of the decay intensity [Eq. 
(52) and Eq. (77) below] is sensitive to this fact as it contains products of the background amplitudes at arbitrary $E$ and $E^{\prime}$.

The amplitude autocorrelation function, Eq. (44), contains two distinct energy dependences, one characterized by $\Gamma_{N}$ which is analogous to Ericson's correlation width as defined by Eq. (71) and another characterized by $\Gamma_{S}+\Gamma^{\downarrow}$ the width of the doorway. Writing Eq. (44) in terms of $x$ and $x^{\prime}$ defined by Eqs. (53) and (54)

$$
c_{00}\left(E, E^{\prime}\right)=\frac{16 i}{\xi} I_{\text {in }}^{\mathrm{av}} I_{\text {in }}^{\mathrm{fl}} \frac{1}{[x+i]^{2}\left[x-x^{\prime}+2 i / \xi\right]\left[x^{\prime}-i\right]^{2}},
$$

we see that it in fact depends only on the ratio $\Gamma_{N} /\left(\Gamma_{S}\right.$ $\left.+\Gamma^{\downarrow}\right)$ and it is through Eq. (44) that this variable enters our calculation of the variance of the decay intensity.

The amplitude autocorrelation function is not an observable quantity. The correlation width, Eq. (71), must be extracted from correlation analysis of the cross section. The cross section autocorrelation function, $C_{a b}\left(E, E^{\prime}\right)$, for cross section $\sigma_{a b}=\left|A_{a b}\right|^{2}$ is defined by

$$
\begin{aligned}
& C_{a b}\left(E, E^{\prime}\right)=\overline{\sigma_{a b}(E) \sigma_{a b}\left(E^{\prime}\right)}-\overline{\sigma_{a b}(E)} \overline{\sigma_{a b}\left(E^{\prime}\right)} \\
& \approx\left|c_{a b}\left(E, E^{\prime}\right)\right|^{2} \\
& +2 \operatorname{Re} \overline{A_{a b}(E)^{*}} c_{a b}\left(E, E^{\prime}\right) \overline{A_{a b}\left(E^{\prime}\right)} \\
& \approx\left[\overline{\sigma_{a b}^{\mathrm{fl}} 2}+2 \sigma_{a b}^{\mathrm{av}} \overline{\sigma_{a b}^{\mathrm{fl}}}\right] \frac{\Gamma^{2}}{\left(E-E^{\prime}\right)^{2}+\Gamma^{2}} .
\end{aligned}
$$

Here, $\sigma_{a b}^{\mathrm{av}}=\left|\overline{A_{a b}}\right|^{2}$ is the background cross section. The fluctuation contribution to the cross section in terms of the transmission coefficients is given by the Hauser-Feshbach formula

$$
\sigma_{a b}^{\mathrm{fl}} \approx \frac{T_{a} T_{b}}{\sum_{c} T_{c}}
$$

or some modification of it designed to account of width fluctuations, direct reactions, etc. [15,20].

Equation (52) for the variance of the decay intensity can be written in terms of the cross section autocorrelation function defined by Eq. (74) as

$$
\overline{\left(\Delta I_{\mathrm{in}}\right)^{2}}=\left(2 \pi \Gamma_{S}\right)^{-2} \int_{-\infty}^{\infty} d E \int_{-\infty}^{\infty} d E^{\prime} C_{00}\left(E, E^{\prime}\right) .
$$

The same comments concerning the energy independence of the background amplitude apply to the derivation of Eq. (75) as applied to the derivation of Eq. (70). Likewise $C_{00}\left(E, E^{\prime}\right)$ in the case of the present paper [the integrand in Eq. (52)] is distinguished from Eq. (75) by its explicit inclusion of the energy dependence of the background. Equation (52) and (74) assume that only pairwise correlations are present. Both
Eqs. (70) and (75) are valid when $\Sigma_{a} T_{a} \gg 1$, that is, in the strongly overlapping resonance region.

\section{B. Expression of the decay intensity and variance in terms of transmission coefficients}

Following GW we introduce two transmission coefficients, $T_{0}(E)$ and $T_{N}$, where

$$
\begin{aligned}
T_{0}(E)=1-\left|\overline{S_{00}}\right|^{2} & =\frac{\Gamma_{S} \Gamma^{\downarrow}}{\left(E-E_{0}\right)^{2}+\left(\Gamma_{S}+\Gamma^{\downarrow}\right) / 4} \\
& =\frac{4 I_{\text {in }}^{\mathrm{av}}\left(1-I_{\mathrm{in}}^{\mathrm{av}}\right)}{4\left(E-E_{0}\right)^{2} /\left(\Gamma_{S}+\Gamma^{\downarrow}\right)+1}
\end{aligned}
$$

describes transmission from the $|Q\rangle$ to the $\mathrm{SD}$ band and

$$
T_{N}=2 \pi \Gamma_{N} / d
$$

describes their transmission to ND states of lower spin. We have not derived Eq. (80). For the purposes of the present paper it can be taken as the definition of $T_{N}$. The reader is referred to the discussion in Sec. VIIIH of Ref. [21] which contrasts the relation of the correlation width $\Gamma$ to transmission coefficients with the the corresponding relation for the average width $\overline{\Gamma_{q}}$.

We have written $T_{0}(E)$ in the form given by Eq. (79) in order to emphasise that it is not simply a function of a single dimensionless variable, the ratio $\Gamma^{\downarrow} / \Gamma_{S}$. It is energy dependent, the energy dependence being characterized by $\Gamma_{S}$ $+\Gamma^{\downarrow}$, the total width of doorway state $|0\rangle$. Only it's maximum $T_{0}\left(E_{0}\right)=4 I_{\text {in }}^{\text {av }}\left(1-I_{\text {in }}^{\text {av }}\right)$ can be expressed solely in terms of $\Gamma^{\downarrow} / \Gamma_{S}$. Thus, a quantity sensitive to the gross energy dependence of $T_{0}(E)$ should depend on $\Gamma_{S}+\Gamma^{\downarrow}$. Writing the average decay intensity Eq. (15) in terms transmission coefficients

$$
\overline{I_{\text {in }}}=1-\left(2 \pi \Gamma_{S}\right)^{-1} \int_{-\infty}^{\infty} d E\left\{T_{0}(E)-2\left[T_{0}(E)\right]^{2} / T_{N}\right\},
$$

we see that it compares the total width of the doorway $|0\rangle$ with the width for the feeding of $|0\rangle$ [thanks to inclusion of the normalization factor $2 \pi \Gamma_{S}$ in the definition of $I_{\text {in }}$ in Eq. (1)]. The variance, Eq. (52), may be written as

$$
\begin{aligned}
& \overline{\left(\Delta I_{\mathrm{in}}\right)^{2}} \\
& \approx\left(2 \pi \Gamma_{S}\right)^{-2} \int_{-\infty}^{\infty} d E \int_{-\infty}^{\infty} d E^{\prime}\left\{\frac{4 T_{0}(E)^{2} T_{0}\left(E^{\prime}\right)^{2}}{\left[2 \pi\left(E-E^{\prime}\right) / d\right]^{2}+T_{N}^{2}}\right. \\
& \left.\quad+4 \operatorname{Im} \frac{\overline{A_{00}(E)} T_{0}(E) T_{0}\left(E^{\prime}\right) \overline{A_{00}\left(E^{\prime}\right)^{*}}}{2 \pi\left(E-E^{\prime}\right) / d+i T_{N}}\right\} .
\end{aligned}
$$

As discussed in Sec. V A and made explicit by Eqs. (59) and (61), the integrand of Eq. (82) which clearly contains 
two characteristic energy scales in fact only depends on their ratio, $\Gamma_{N} /\left(\Gamma_{S}+\Gamma^{\downarrow}\right)$, the ratio of the correlation width to the doorway width.

Equation (41) may also be expressed in terms of the transmission coefficients $T_{0}(E)$ and $T_{N}$. Using Eq. (43) for $\Gamma^{\uparrow}$ we get

$$
\Gamma=\frac{d}{2 \pi} T_{N}\left[1-\frac{T_{0}(E) / T_{N}}{I_{\mathrm{in}}^{\mathrm{av}}}\right],
$$

so that the neglect of $\Gamma^{\uparrow}$ in $\Gamma$ is justified when $T_{0}(E) / T_{N}$ $\ll I_{\text {in }}^{\text {av }} \leqslant 1$. Let us also write the correlation length $\xi$, Eq. (56), in terms of the transmission coefficients

$$
\xi=\left(2 \pi / T_{N}\right) \frac{\Gamma_{S}+\Gamma^{\downarrow}}{d} .
$$

In the case of compound nucleus scattering, extraction of $\Gamma$ from a measurement of cross section autocorrelation function, using say Eq. (75), permits the determination of the density of compound nucleus states $1 / d$ by application of Eq. (71) [22]. A more recent example of energy-autocorrelation analysis may be found in Ref. [23] where fluctuations in dissipative binary heavy ion collisions are studied. In the present case of the decay out of a superdeformed band extraction of $\xi$ from the variance of the intensity, permits the determination of the ratio $\left(\Gamma_{S}+\Gamma^{\downarrow}\right) / \Gamma_{N}$, or, given $T_{N}$ (equivalently $\left.\Gamma_{N} / d\right)$ determination of the ratio $\left(\Gamma_{S}\right.$ $\left.+\Gamma^{\downarrow}\right) / d$.

\section{Comparison with the results of $\mathrm{Gu}$ and Weidenmüller}

GW also take inspiration from statistical nuclear reaction theory but use the MPI approach [24]. The MPI approach is concerned with the analytic calculation of ensemble averages, a procedure which is equivalent to the calculation of energy averages. Reference [24] use the supersymmetry method of calculating ensemble averages to derive an exact expression for $\overline{S_{a b}^{\mathrm{fl}}(E) S_{c d}^{\mathrm{fl}}\left(E^{\prime}\right)}$. Their result is found to be expressible in terms of the difference of the two energies, $E-E^{\prime}$, and transmission coefficients. The transmission coefficients themselves are expressed as functions of $(E$ $\left.+E^{\prime}\right) / 2$. The relationship between the results of [15-17] and those of Ref. [24] is discussed in Refs. [20,25]. Several results of Refs. [15-17] can be obtained from that of Ref. [24] by expanding in powers of the transmission coefficients or inverse powers of the sum of the transmission coefficients [20].

Calculation of the average of the fluctuation intensity requires the energy integral of the average of the product of two $S$-matrix elements at the same energy. GW use the results of Ref. [24] for $\overline{\left|S_{00}^{\mathrm{fl}}(E)\right|^{2}}$ to calculate the average decay intensity. As was already noted in Sec. III, GW include the energy dependence of the background amplitude characteristic of an isolated doorway resonance in their calculation by using the energy dependent transmission coefficient $T_{0}(E)$, Eq. (78), in their Eq. (24) for $\overline{I_{\mathrm{in}}^{\mathrm{H}}}$. The fact that we use the same energy dependence as GW for the background is re- sponsible for the agreement we obtain with GW concerning $\overline{I_{\text {in }}}$ 's dependence on $\Gamma^{\downarrow} / \Gamma_{S}$. The differences between our results and those of GW for the decay intensity stem from the assumptions we make which restrict our results to $\Gamma_{N} / d$ $\gg 1$.

Calculation of the variance of the intensity requires the four-point function at two energies integrated over both energies, that is, it requires $\overline{S_{00}^{\mathrm{fl}}(E) S_{00}^{\mathrm{fl}}(E) * S_{00}^{\mathrm{fl}}\left(E^{\prime}\right) S_{00}^{\mathrm{fl}}\left(E^{\prime}\right)^{*}}$ integrated over $E$ and $E^{\prime}$. Calculation of the four-point function at two energies was carried out using the supersymmetry method in Ref. [26]. Their result, like that of Ref. [24] for the two-point function depends explicitly only on $E-E^{\prime}$ and the transmission coefficients which are again expressed as functions of $\left(E+E^{\prime}\right) / 2$. Within the assumption that only pairwise correlations are important, as was assumed in Eqs. (52) and (74), the two-point function is enough to calculate the variance. Reference [20] showed numerically that the exact expression of Ref. [24] specialized to the amplitude autocorrelation function confirms the correctness of Eq. (70) in the region of strongly overlapping resonances. However, unlike Eq. (70), the amplitude autocorrelation function as given by Eq. (44) depends on the background amplitude at two different energies, that is, it depends on $\overline{A_{00}(E)}$ and $\overline{A_{00}\left(E^{\prime}\right)}$. When $E=E^{\prime}$ it reduces to Eq. (45) which can be expressed in terms of the transmission coefficients $T_{0}(E)$ and $T_{N}$. Thus the decay intensity can be expressed in terms of these transmission coefficients as was done in Eq. (81). The applicability of Ref. [24] to calculation of the decay intensity owes itself to the fact that the decay intensity may be expressed in terms of transmission coefficients.

Equation (44) cannot be written in terms of $T_{0}([E$ $\left.\left.+E^{\prime}\right] / 2\right)$ and the same applies to the variance as is apparent from Eq. (82). Thus it is not clear whether Ref. [24] serves as a means to obtain results corresponding to Eqs. (44) and (82) which are valid for arbitrary $\Gamma_{N} / d$. It would be an interesting challenge to derive an expression for the variance which could be used for any value of $\Gamma_{N} / d$ since for the regions which have been most frequently studied experimentally [2], the $A \approx 150$ and $A \approx 190$ regions, $\Gamma_{N} / d \ll 1$.

GW do not use the supersymmetry method to calculate the variance. They instead estimate the variance by performing a numerical simulation. The analytic structure of the variance was not investigated in GW and their results make no reference to the variable $\Gamma_{N} /\left(\Gamma_{S}+\Gamma^{\downarrow}\right)$. Given the close resemblance of the conclusions about the analytic structure of the decay intensity which may be inferred from the exact result of GW and our approximate result for $\Gamma_{N} / d \gg 1$ it seems probable that the dependence of the variance on $\Gamma_{N} /\left(\Gamma_{S}+\Gamma^{\downarrow}\right)$ which we have found for $\Gamma_{N} / d \gg 1$ persists for arbitrary $\Gamma_{N} / d$.

\section{CONCLUSIONS}

In conclusion, we have derived analytic formulas for the energy average and variance of the intraband decay intensity of a superdeformed band in terms of variables which usefully describe the decay out. The formulas given by Eq. (47) for the fluctuation contribution to the average intensity $\overline{I_{\text {in }}^{\mathrm{fl}}}$ and by 
Eq. (55) for the variance $\overline{\left(\Delta I_{\text {in }}\right)^{2}}$ were derived by making assumptions and approximations which are strictly valid only in the strongly overlapping resonance region, $\Gamma_{N} / d \gg 1$. However, these formulas are seen from Figs. 2 and 3 to work well when $\Gamma_{N} / d=1$ and provide a qualitative description even when $\Gamma_{N} / d=0.1$. This means that Eq. (47) and Eq. (55) cannot be applied to the mass 150 and 190 regions where $\Gamma_{N} / d \sim 0.001$ but they may prove themselves of practical use in other mass regions. In any case our results clarify the analytic structure of the results obtained by GW. In particular we have revealed that the variance of the decay inten- sity depends on the correlation length $\Gamma_{N} /\left(\Gamma_{S}+\Gamma^{\downarrow}\right)$ in addition to the two dimensionless variables $\Gamma^{\downarrow} / \Gamma_{S}$ and $\Gamma_{N} / d$ on which the average of the decay intensity depends. Measurement of the variance of the decay intensity could yield the mean level density of the ND states in analogy with autocorrelation analysis of cross sections.

\section{ACKNOWLEDGMENTS}

This work was partially supported by FAPESP.
[1] T. Lauritsen et al., Phys. Rev. Lett. 88, 042501 (2002).

[2] A. Lopez-Martens et al., Phys. Scr. T88, 28 (2000).

[3] A. Dewald et al., Phys. Rev. C 64, 054309 (2001).

[4] R. Krucken, A. Dewald, P. von Brentano, and H.A. Weidenmuller, Phys. Rev. C 64, 064316 (2001).

[5] E. Vigezzi, R.A. Broglia, and T. Dossing, Phys. Lett. B 249, 163 (1990); Nucl. Phys. A520, 179c (1990).

[6] Y.R. Shimizu, F. Barranco, R.A. Broglia, T. Dossing, and E. Vigezzi, Phys. Lett. B 274, 253 (1992).

[7] Y.R. Shimuzu, E. Vigezzi, T. Dossing, and R. Broglia, Nucl. Phys. A557, 99c (1993).

[8] Y.R. Shimizu, M. Matsuo, and K. Yoshida, Nucl. Phys. A682, 464c (2001).

[9] K. Yoshida, M. Matsuo, and Y.R. Shimizu, Nucl. Phys. A696, 85 (2001).

[10] S. Aberg, Phys. Rev. Lett. 82, 299 (1999); Nucl. Phys. A649, 392c (1999).

[11] R. Krucken, Phys. Rev. C 62, 061302(R) (2000).

[12] A.J. Sargeant, M.S. Hussein, M.P. Pato, N. Takigawa, and M. Ueda, Phys. Rev. C 65, 024302 (2002)

[13] J.z. Gu and H.A. Weidenmuller, Nucl. Phys. A660, 197 (1999).
[14] H.A. Weidenmuller, P. von Brentano, and B.R. Barrett, Phys. Rev. Lett. 81, 3603 (1998).

[15] H. Feshbach, Theoretical Nuclear Physics: Nuclear Reactions (Wiley, New York, 1992), Chap. IV.

[16] M. Kawai, A.K. Kerman, and K.W. McVoy, Ann. Phys. (N.Y.) 75, 156 (1973).

[17] T. Ericson, Ann. Phys. (N.Y.) 23, 390 (1963).

[18] P. R. Wallace, Mathematical Analysis of Physical Problems (Dover, New York, 1984), Chap. VIII.

[19] J. Richert, M.H. Simbel, and H.A. Weidenmuller, Z. Phys. A 273, 195 (1975).

[20] J.J. Verbaarschot, Ann. Phys. (N.Y.) 168, 368 (1986).

[21] T.A. Brody, J. Flores, J.B. French, P.A. Mello, A. Pandey, and S.S.M. Wong, Rev. Mod. Phys. 53, 385 (1981).

[22] T. Ericson and T. Mayer-Kuckuk, Annu. Rev. Nucl. Sci. 16, 183 (1966)

[23] M. Papa et al., Phys. Rev. C 61, 044614 (2000).

[24] J.J. Verbaarschot, H.A. Weidenmuller, and M.R. Zirnbauer, Phys. Rep. 129, 367 (1985).

[25] Y. Takahashi and S. Yoshida, Nucl. Phys. A507, 371 (1990).

[26] E.D. Davis and D. Boose, Z. Phys. A 332, 427 (1989). 